\title{
Topographic Instability: Tests
}

\author{
JoSEPH EGGer \\ Meteorologisches Institut der Universität Munich, Munich, Germany \\ KLAus-Peter HoinKa \\ DLR, Institut für Physik der Atmosphäre, Oberpfaffenhofen, Germany
}

(Manuscript received 4 October 2006, in final form 16 May 2007)

\begin{abstract}
Theories of topographic instability predict growth of perturbations of mean flow and wave modes due to their interaction with mountains under favorable conditions. Mountain torques form an important part of this interaction. It has been suggested that topographic instabilities contribute significantly to the subseasonal variability of the atmosphere but observational tests of topographic instability mechanisms have not yet been performed. Greenland is selected as a test bed because of its isolation, simple shape, and appropriate size. The observed flow development during mountain torque events is investigated in terms of a regression analysis. Changes of axial angular momentum and zonal mean wind with respect to the torques are monitored for domains covering Greenland since the acceleration (deceleration) of the regional zonal flow in response to a positive (negative) torque is a key feature of topographic instability. In particular, southern and northern analysis domains are considered separately in order to test "dipole" instability theories in addition to "monopole" situations where the meridional extent of the pressure perturbations is similar to that of Greenland. Moreover, zonal bands are used as analysis domains. It is found that the response of the zonal wind to the torques is quite small and not systematic. There is no evidence of monopole or dipole topographic instability. A less detailed analysis for the Tibetan Plateau leads to the same result. Reasons for these negative outcomes are discussed as are shortcomings of the tests.
\end{abstract}

\section{Introduction}

Charney and DeVore (1979, hereafter CDV) found a new instability when analyzing a low-order model of barotropic channel flow over topography with multiple equilibria. This topographic instability is associated with an equilibrium state in the presence of orography, zonal mean flow forcing, and surface friction (see also Ghil and Childress 1987). As outlined, for example, by Jin and Ghil (1990), the basic mechanism of this instability is simple. A stationary high resides above a mountain in westerly "superresonant" mean zonal flow where free Rossby waves of the mountain's scale would propagate eastward (Fig. 1) in the channel. The high is in phase with the mountain in Fig. 1 because the flow is assumed frictionless. Correspondingly there is no

Corresponding author address: Joseph Egger, Meteorologisches Institut der Universität Munich, Theresienstr. 37, 80333 Munich, Germany.

E-mail: j.egger@lrz.uni-muenchen.de

DOI: $10.1175 / 2007$ JAS2311.1

(C) 2008 American Meteorological Society mountain torque exerted [see (2.1) and the related discussion]. Assume now an eastward shift of the high due to some perturbation. This displacement leads to a reduction of surface pressure at the windward side and an increase in the lee, so that a positive axial torque is exerted on the atmosphere. This torque accelerates the westerly flow in the mountain region. This leads to eastward advection of the high and further surface pressure increase in the lee under favorable conditions. Hence, the torque is growing and we have an instability that is particularly effective near resonance when the mountain scale is close to the stationary Rossby wavelength. There is no instability in the "subresonant" case where a trough resides above the mountain.

Further theories of topographic instability evolved quickly after the seminal contribution by CDV. The baroclinic case was first considered by Charney and Straus (1980) for channel flow. Pedlosky (1981) included nonlinear effects. The horizontal resolution of the models has been enhanced as well. Jin and Ghil (1990) investigated barotropic topographic instability 


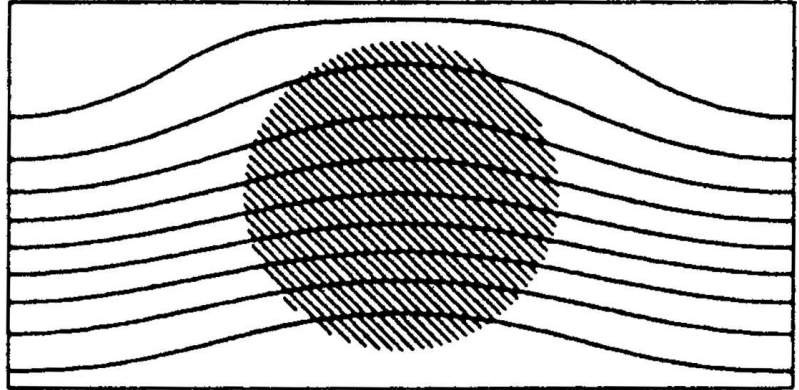

FIG. 1. Schematic of the superresonant situation in barotropic stationary $\beta$-plane channel flow over circular topography (shaded). Streamlines are bold. Adapted from Jin and Ghil (1990).

for more general basic flows than admitted by CDV. They found a dipole topographic instability that favors a meridional pressure dipole on the windward side and in the lee while the CDV instability works with a monopole. There is almost no mean torque in these cases but there is appreciable form drag in the southern half that is of opposite sign to that in the northern part. Such distributions of the form drag can, however, also occur for streamfunction patterns that one would classify as monopole perturbations (Fyfe and Derome 1986). The step to the spherical domain has been made by Egger and Metz (1981), who demonstrated on the basis of a severely truncated barotropic model that the superrotational mean flow component $u_{o} \cos \varphi$ will tend to an equilibrium value $\left\langle u_{o}\right\rangle \approx 0$ in the presence of mountains and in the absence of friction. Frederiksen and Frederiksen (1991) solved this problem more generally by using methods of statistical mechanics. There is an upper limit for $\left\langle u_{o}\right\rangle$ such that all Rossby wave modes of the flow have a westerly phase speed. That implies indeed $\left\langle u_{o}\right\rangle \approx 0$. Topographic instability is presumably rare in this statistical equilibrium because all flow modes are subresonant with respect to $\left\langle u_{o}\right\rangle$. Frederiksen (1982) and Frederiksen and Carnevale (1986) demonstrated that eastward solid body rotation is always unstable in the presence of topography. The time scale of the adjustment of $\mathrm{u}_{\mathrm{o}}$ to the limiting value is, however, fairly long for realistic topography and mean winds. Revell and Hoskins (1984) analyzed baroclinic topography instability on the sphere on the basis of the primitive equations. Their linear stability analysis led them to conclude that topographic instability is presumably too weak to be detectable in the atmosphere. Frederiksen and Bell (1987) presented a stability analysis of the Northern Hemisphere winter mean flow using a fivelevel quasigeostrophic model where they paid special attention to topographic effects. They found that incorporation of realistic topography reduces the growth rate of most modes. The topographic impact is largest for slowly growing equivalent barotropic modes. There do not appear, however, to be cases of "pure" topographic instability, which disappear if the topography is removed.

Verification of the theoretical results did not develop at the same pace as the ramification of the theories. Although there is a rich literature on the relation of mountain torques and atmospheric variability (e.g., Weickmann 2003; Lott et al. 2004), the issue of verification of topographic instability theories did not attract much attention. Tung and Rosenthal (1985) had a critical look at the CDV model and some of its relatives but did not embark on data analysis. Metz (1985) came closest to such tests by considering the relation of blocking and mountain torque in observations in light of the predictions of the CDV model. He found negative values of the form drag at mid- and high latitudes at the early stages of blocking when the zonally averaged wind exhibits negative anomalies. Dickey et al. (1991) report on GCM experiments with and without topography where a spectral peak near periods of 40 days is found in the topography run only. These authors suggest that the related oscillations are associated with the instabilities analyzed by Jin and Ghil (1990).

It is the purpose of this paper to provide the first tests of the topographic instability mechanism on the basis of observations. The strategy of the tests is discussed in section 2. The tests are presented in section 3 .

\section{Tests: Strategy}

It is clear from the literature quoted above that events of topographic instability involve by definition regional mountain torques,

$$
T_{o}=-\int_{F_{o i}} p_{s} \frac{\partial h}{\partial \lambda} a^{2} \cos \varphi d \varphi d \lambda,
$$

where $p_{s}$ is surface pressure, $h$ is topography, and the area $F_{o i}$ covers in most cases the mountain. The torque (2.1) affects the axial angular momentum of the atmosphere, and thus, the zonal winds. Torques will be evaluated for areas $F_{o i}$ as well, which do not fully cover the mountain. That is necessary for tests of the dipole instability.

It is, however, not so clear how to choose the domain of response of volume $V_{i}$ and area $F_{i}$ wherein the zonal wind is expected to react to the torque. This choice is trivial in $\beta$-plane models where $V_{i}$ is simply the volume of the channel atmosphere. In reality the global atmosphere appears to be the appropriate choice but, as will be shown quantitatively below, the related global wind 
changes are too small to be of interest. Moreover, the dynamics of topographic instability as briefly outlined above rely on advective processes near the mountain. The zonal flow has to change in the mountain area to have a sufficiently strong impact. On the other hand, the condition of closeness to superresonance may not be that important. What is needed is a substantial climatological anticyclone in the mountain's region. Nevertheless, there is substantial arbitrariness in the choice of the response volume. We try to overcome this problem by admitting several analysis areas $F_{i}$ that scan a wide range of zonal scales to be detailed below.

So far, theories of topographic instability have been formulated mainly within the quasigeostrophic framework where the mountain torque affects exclusively the mean zonal flow. This leads to an overestimation of growth rates (Egger 2003). In the atmosphere, it is necessary to consider the axial angular momentum

$$
M_{i}=\int_{v_{i}} \rho(u+\Omega a \cos \varphi) a \cos \varphi d V
$$

of the analysis domain because torques are factors in the angular momentum balance. In (2.2), $\rho$ is the density, $u$ is the zonal wind component, and $\Omega=2 \pi$ day $^{-1}$. The effect of the mountain torque on the zonal wind can be singled out by analyzing separately the wind term

$$
M_{w i}=\int_{v_{i}} \rho u a \cos \varphi d V .
$$

It is, of course, also of interest to monitor the mass term $M_{m i}$, which represents the contribution of the earth's rotation velocity to $M_{i}$ in (2.2). Although mountain torques do not affect directly the mass term, conversions between wind and mass term are possible via the Coriolis term in the zonal momentum equation so that part of the angular momentum generated by the torque may alter the mass term.

Although the global axial angular momentum is affected by mountain and friction torques only, this is not so for our specific domains $V_{i}$ on the sphere where the budget equation is

$$
\begin{aligned}
\frac{d M_{i}}{d t}= & - \text { boundary fluxes }+ \text { pressure torque } \\
& +T_{o i}+T_{f i} .
\end{aligned}
$$

The boundary fluxes comprise fluxes of angular momentum through the boundaries of the domain except the surface. The pressure torque reflects pressure differences between the eastern and western boundary. In addition to the mountain torque $T_{o i}$, there is the friction torque $T_{f i}$, which however, not be considered in the remainder. It is by no means certain that a positive mountain torque will lead to an increase of $M_{w i}$. The other terms in (2.4) are not necessarily small and may override the impact of the mountain torque. Theories of topographic instability in channel flow exclude boundary fluxes and the pressure torque through the specification of the model's boundary conditions. In that case, it is guaranteed that a positive torque leads to an acceleration of the zonal flow.

It is, however, clear despite all these complications that topographic instability events involve the acceleration of regional zonal flows due to the torque exerted by the selected mountain. Tests of this key mechanism of topographic instability will be conducted on a statistical basis. We denote by $C(b, c \mid \tau)$ the covariance of the variables $b$ and $c$ at lag $\tau$ where $b$ leads $c$. Then we have to evaluate $C\left(T_{o i}, M_{w j} \mid \tau\right)$, where the indices $i$ and $j$ refer to the specific analysis domains chosen. There is supporting evidence for topographic instability, if the response of the regional wind term to the torque is positive and sufficiently strong. If not, topographic instability is essentially ruled out.

Tests will be mainly conducted with respect to Greenland. This massif appears to be almost ideally suited for such tests, because it is isolated from other mountain chains and its shape resembles those prescribed in theoretical work. Moreover, the standard deviation of wintertime torques is 7 hadleys $(\mathrm{Ha})$ for Greenland (Table 2; $1 \mathrm{Ha}=10^{18} \mathrm{~J}$ ). This value suggests that a mountain torque event may have substantial impact on regional zonal flows. Assume a control area $F_{i}$ of meridional width $\Delta \varphi=30^{\circ}$ centered at $65^{\circ} \mathrm{N}$ and a 10-Ha torque exerted for, say, 3 days (see also Table 1). Assume, moreover, that there is no transfer of angular momentum to the mass term during that time and that all terms on the right-hand side of (2.4) are negligible except for the mountain torque. The increase of the zonal mean flow in this domain would be 7 (1.6) $\mathrm{m} \mathrm{s}^{-1}$ within these 3 days if the zonal extent of the control volume is $\Delta \lambda=80^{\circ}\left(360^{\circ}\right)$. That is a large change, which should be easily detectable at least for the smaller domain $\left(\Delta \lambda=80^{\circ}\right)$. The choice of Greenland is further motivated by the results of Frederiksen and Bell (1987), who found many unstable global modes that are active in the Greenland region.

Data from the 40-yr European Centre for MediumRange Weather Forecasts (ECMWF) Re-Analysis (ERA-40) set (Uppala et al. 2005) are used to regress the regional angular momentum onto the axial mountain torque exerted by Greenland. The spatial resolution is $1.125^{\circ} \times 1.125^{\circ}$ in the horizontal and $1000 \mathrm{~m}$ in 


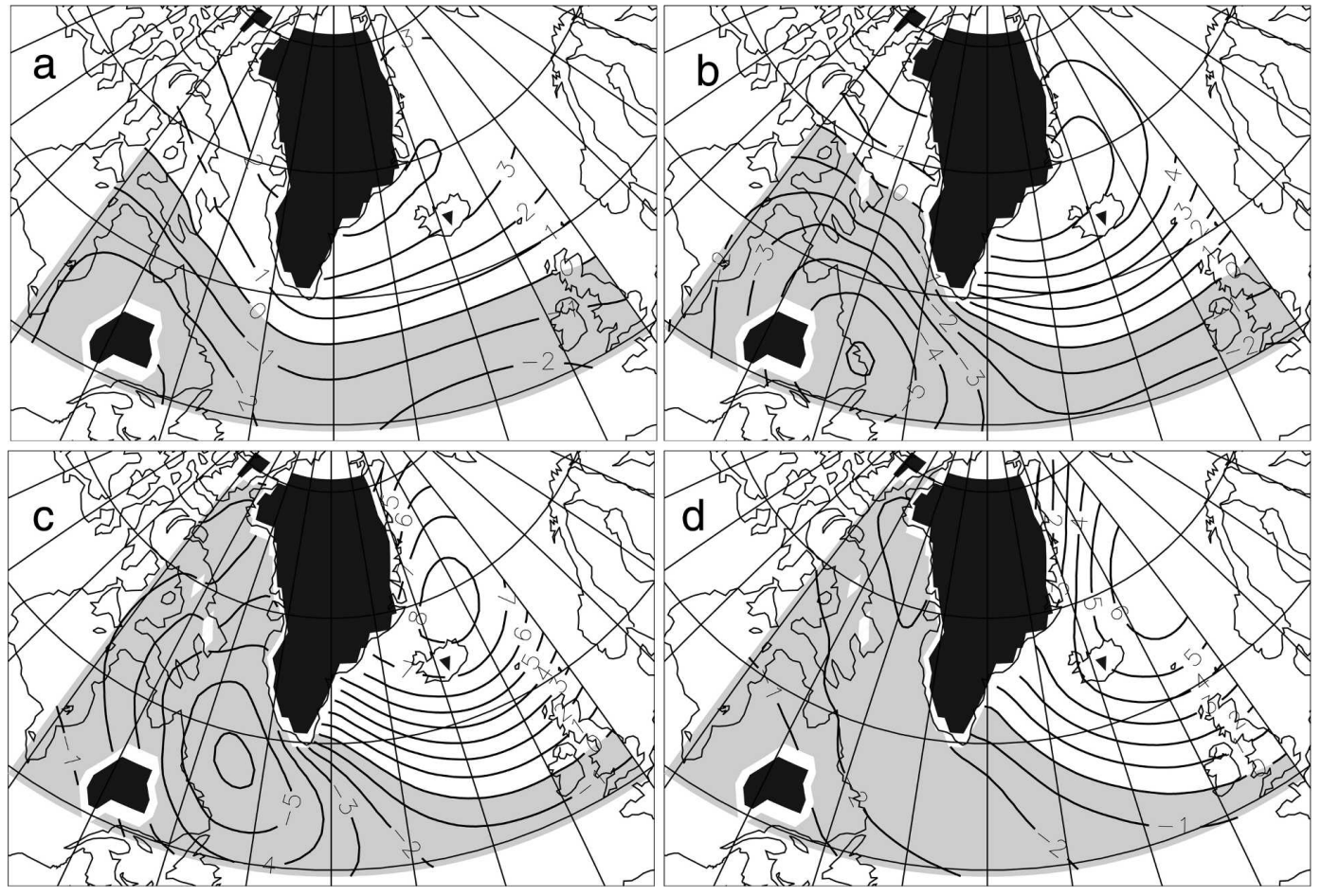

FIG. 2. Cross covariance $C\left(T_{o 1}, p \mid \tau\right)$ of the normalized Greenland winter mountain torque at the height $z=500$ $\mathrm{m}$ with the pressure field at (a) $\tau=-2$, (b) $\tau=-1$, (c) $\tau=0$, and (d) $\tau=1$ day; isolines are restricted to the analysis domain $F_{1}$ (see also Table 1 ). The torque $T_{o 1}$ is normalized with respect to its std dev so that units are hPa with a contour interval of $1 \mathrm{hPa}$.

the vertical with 13 layers. Daily covariance values $C\left(T_{o i}, M_{w j} \mid \tau\right)$ are available for lags $-5 \leq \tau \leq 5$ days. In addition, all data have been low-pass filtered as in Blackmon and Lau (1980) to retain only those oscillations with periods longer than 10 days. This way we hope to shed some light on the role of topographic instability in low-frequency variability.

Further tests will be performed with respect to the Tibetan Plateau. This is a zonally elongated massif of enormous size. Nevertheless, the standard deviation of the winter torques is also about $10 \mathrm{Ha}$. Since the control volume $V_{i}$ must be larger than for Greenland, changes of the zonal wind due to the mountain torque are automatically smaller than those in the Greenland case. Topographic instability near the Tibetan Plateau is, therefore, less likely than near Greenland. For this reason, tests will be less detailed than for Greenland.

\section{Results}

\section{a. Greenland}

Let us first look briefly at typical torque events near Greenland. In winter, the time mean vorticity distribution in the Greenland area shows weak cyclonic vorticity west of the massif and a rather large center of anti- cyclonic vorticity above and to the east of Greenland (not displayed). Although this pattern is not as symmetric as in Fig. 1, there is quite some similarity and, therefore, good reason to expect positive results of the tests. Enhanced westerlies transport more anticyclonic vorticity into the lee. A typical torque event is presented in Fig. 2 where the covariance of the pressure perturbation at a height $z=500 \mathrm{~m}$ with the normalized Greenland torque is shown for several lags. The event is initialized by a low pressure perturbation southwest of Greenland that moves toward the southeast. This perturbation induces a positive torque that is, however, greatly enhanced by the growth of a high in the lee of Greenland. The slow initial motion of the high in the lee fits the predictions of linear topographic instability theory where the unstable modes tend to have vanishing phase speed (e.g., Pedlosky 1981). On the other hand, the upstream development in Fig. 2 is not reproduced by available theories. Nevertheless, the overall sequence of events appears to fit the scenario of monopole topographic instability reasonably well. What has to be demonstrated, however, is the impact of the torque on the regional zonal flow. Does the regional wind term respond positively to the Greenland torque? If so, are the changes large enough? 
TABLE 1 . Analysis domains used in the tests whereby $F_{2}\left(F_{5}\right)$ covers the southern part of $F_{1}\left(F_{4}\right)$ and $F_{3}\left(F_{6}\right)$ covers the northern part (see also Fig. 2).

\begin{tabular}{lrl}
\hline \hline$F_{1}$ & $81^{\circ}, 6^{\circ} \mathrm{W}-0.6^{\circ} \mathrm{E}$ & $48.9^{\circ}-81.6^{\circ} \mathrm{N}$ \\
$F_{1 z}$ & $180^{\circ} \mathrm{W}-180^{\circ} \mathrm{E}$ & $48.9^{\circ}-81.6^{\circ} \mathrm{N}$ \\
$F_{2}$ & $180^{\circ} \mathrm{W}-180^{\circ} \mathrm{E}$ & $48.9^{\circ}-70.3^{\circ} \mathrm{N}$ \\
$F_{3}$ & $180^{\circ} \mathrm{W}-180^{\circ} \mathrm{E}$ & $70.3^{\circ}-81.6^{\circ} \mathrm{N}$ \\
$F_{4}$ & $75^{\circ}, 9^{\circ}-14.1^{\circ} \mathrm{W}$ & $59.1^{\circ}-81.6^{\circ} \mathrm{N}$ \\
$F_{4 z}$ & $180^{\circ} \mathrm{W}-180^{\circ} \mathrm{E}$ & $59.1^{\circ}-81.6^{\circ} \mathrm{N}$ \\
$F_{5}$ & $180^{\circ} \mathrm{W}-180^{\circ} \mathrm{E}$ & $59.1^{\circ}-70.3^{\circ} \mathrm{N}$ \\
$F_{6}$ & $180^{\circ} \mathrm{W}-180^{\circ} \mathrm{E}$ & $70.3^{\circ}-81.6^{\circ} \mathrm{N}$ \\
\hline
\end{tabular}

Dipole instability is certainly not represented in Fig. 2. That is not surprising because the total mountain torque of Greenland would be small during such events. A covariance analysis based on the total torque will miss such events automatically.

The various analysis domains $F_{i}$ used in the tests are defined in Table 1 and displayed in Fig. 3. The test area $F_{1}$ covers part of Canada, Greenland, and parts of the North Atlantic as in Egger and Hoinka (2006). There is also a southern part $F_{2}$ and a northern $F_{3}$ so that $F_{1}=$ $F_{2}+F_{3}$. These subdomains are introduced in order to capture eventual events of dipole instability. In addition, a smaller regional domain $F_{4}$ is considered as well, which essentially covers Greenland, again with a partitioning $F_{5}, F_{6}$. The zonal domains $F_{1 z}$ and $F_{4 z}$ extend around the globe.

The channel length $W$ in CDV and Pedlosky (1981) is double the width of the mountain and always larger than that in later work (see also Fig. 1). In that sense, $F_{1}$ is kind of a minimum size with $W \approx 4000 \mathrm{~km}$. The even smaller domain $F_{4}$ has been chosen on grounds that the impact of the torques may be better visible if the con-

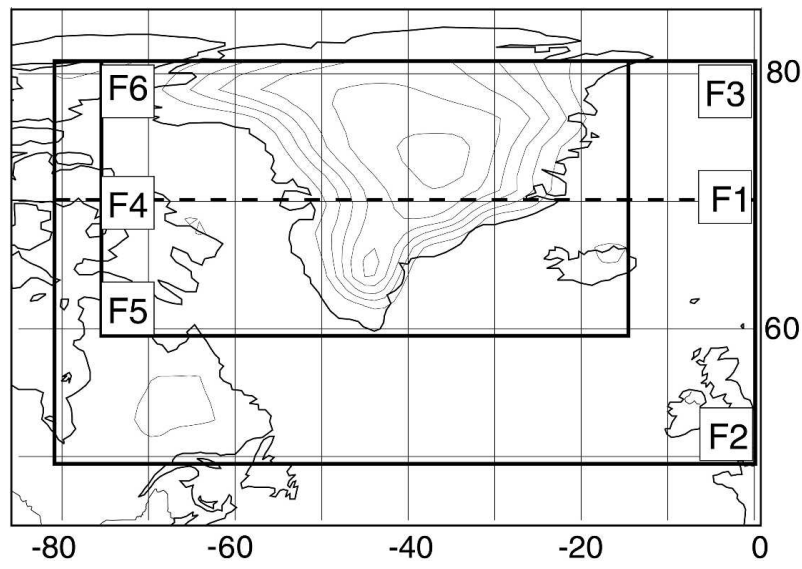

FIG. 3. Domains $F_{1}-F_{6}$ covering Greenland as used in the tests; see also Table 1 . The mountain torques for $F_{4}, F_{5}$, and $F_{6}$ are the same as for $F_{1}, F_{2}$, and $F_{3}$.
TABLE 2. Std dev of the mountain torque $T_{o i}(\mathrm{Ha})$ for the domains $F_{1}-F_{3}$ and all seasons; the results for the filtered series are given in parentheses. The torques for $F_{4}-F_{6}$ agree with those for $F_{1}-F_{3}$.

\begin{tabular}{lcccc}
\hline \hline & $\begin{array}{c}\text { March-May } \\
\text { (MAM) }\end{array}$ & $\begin{array}{c}\text { June-August } \\
\text { (JJA) }\end{array}$ & $\begin{array}{c}\text { September- } \\
\text { October } \\
\text { (SON) }\end{array}$ & $\begin{array}{c}\text { December- } \\
\text { January } \\
\text { (DJ) }\end{array}$ \\
\hline$F_{1}$ & $5.3(3.8)$ & $3.5(2.4)$ & $5.5(3.7)$ & $6.7(4.8)$ \\
$F_{2}$ & $3.5(2.6)$ & $2.2(1.5)$ & $3.5(3.1)$ & $4.3(2.5)$ \\
$F_{3}$ & $2.4(1.7)$ & $1.7(1.2)$ & $2.4(1.7)$ & $3.1(1.8)$ \\
\hline
\end{tabular}

trol volumes are small. Since the angular momentum $M_{1}$ in $V_{1}$ experiences the same torque as $M_{4}$, the response of the zonal wind in the smaller volume $V_{4}$ might be much larger. The domains $F_{1 z}$ and $F_{4 z}$ represent the maximum extent.

The Greenland torques are calculated such that only the topography of Greenland enters (see Egger and Hoinka 2006 for details). The torques for $F_{1}$ and $F_{4}$ are identical as are those for the pairs $F_{2}, F_{5}$ and $F_{3}, F_{6}$.

The standard deviations of the mountain torques $T_{o i}$ are given in Table 2 for the domains $F_{1}-F_{3}$ and for all seasons. The torques peak in winter and are smallest in summer. By and large, the sum of the standard deviations for $F_{2}$ and $F_{3}$ equals that of $F_{1}$. The seasonal mean values are in the range 1.0-2.2 Ha for $F_{1}, 0.4-0.7 \mathrm{Ha}$ for $F_{2}$, and $0.4-1.5 \mathrm{Ha}$ for $F_{3}$ so that the contribution of the northern part to the mean is larger than that of the southern one while it is the reverse for the standard deviations. The standard deviations of the filtered time series amount to more than half the values of the unfiltered data. The autocorrelation of $T_{o 1}$ decays fairly quickly as is typical of mountain torques (Fig. 4a). This rapid decay reflects, of course, the fast motion of the synoptic systems in Fig. 2. There is little seasonal variation except that there appears to be no zero-crossing in winter. The autocorrelations of the filtered torques decay more slowly, of course, but with fairly distinct zerocrossings for $\tau \approx 7$ days (Fig. $4 \mathrm{~b}$ ).

The correlation coefficient of the torques $T_{o 2}$ and $T_{o 3}$ is $\sim 0.6$ both in winter and summer. Prevalence of dipole situations would lead to negative correlations. There is rapid decay, of course, of the cross correlation of $T_{o 2}$ and $T_{o 3}$ with increasing lag $|\tau|$. The autocorrelations of $T_{o 2}$ and $T_{o 3}$ are fairly similar to those in Fig. 4.

The seasonal variation of the standard deviations of the mass and wind terms is surprisingly small. For example, the standard deviation of $M_{w 1}$ varies between 1.2 and $1.5 \times 10^{6} \mathrm{Ha} s$ with a maximum in fall. The standard deviation of the mass term is about one-third that of the wind term. This ratio is quite similar to that 


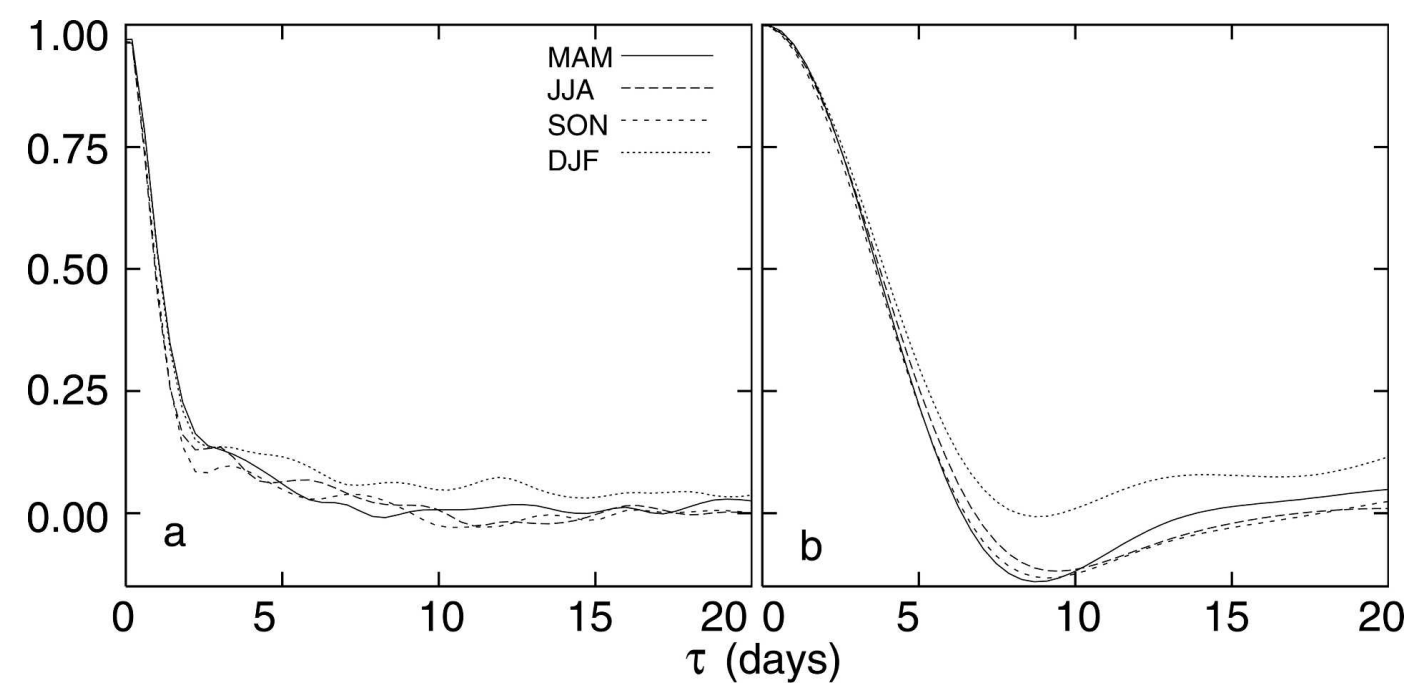

FIG. 4. Autocorrelation of the mountain torques $T_{o 1}$ of Greenland for all seasons as a function of lag in days for (a) unfiltered and (b) low-pass-filtered data.

in the global case (Weickmann et al. 2000). The standard deviations for $F_{2}\left(F_{5}\right)$ are fairly close to those of $F_{1}$ $\left(F_{4}\right)$ both for the wind and mass term while that for the northern part $F_{3}\left(F_{6}\right)$ is substantially smaller. The same is true for the mean values. The standard deviations of the filtered wind and mass terms are fairly close to those of the unfiltered ones.

The autocorrelations of the wind terms for the domains $F_{4}-F_{6}$ (Fig. 5) in fall decay more slowly than those of the torques just as in the global atmosphere. This is true for all seasons and also for the domains $F_{1}-F_{3}$. The corresponding autocorrelations for the zonal belts $F_{i z}$ (Fig. 5b) decay more slowly. Wind terms can be transformed easily into estimates of the zonal mean flow

$$
\hat{u}_{i}=M_{w i} A^{-1}
$$

with $A_{i}=\int_{v_{i}} \rho_{o} a \cos \varphi d V$ where $\rho_{\mathrm{o}}$ is a reference density; the hat stands for the zonal mean. For example, $A_{1}$ $=3.8 \times 10^{5} \mathrm{Ha} \mathrm{s}^{2} \mathrm{~m}^{-1}$ and $A_{3}=6 \times 10^{4} \mathrm{Ha} \mathrm{s}^{2} \mathrm{~m}^{-1}$. The autocorrelations of the mass terms decay somewhat faster than those of the wind terms (Fig. 6), at least as far as $F_{4}$ and $F_{5}$ are concerned. The mass term pertur-

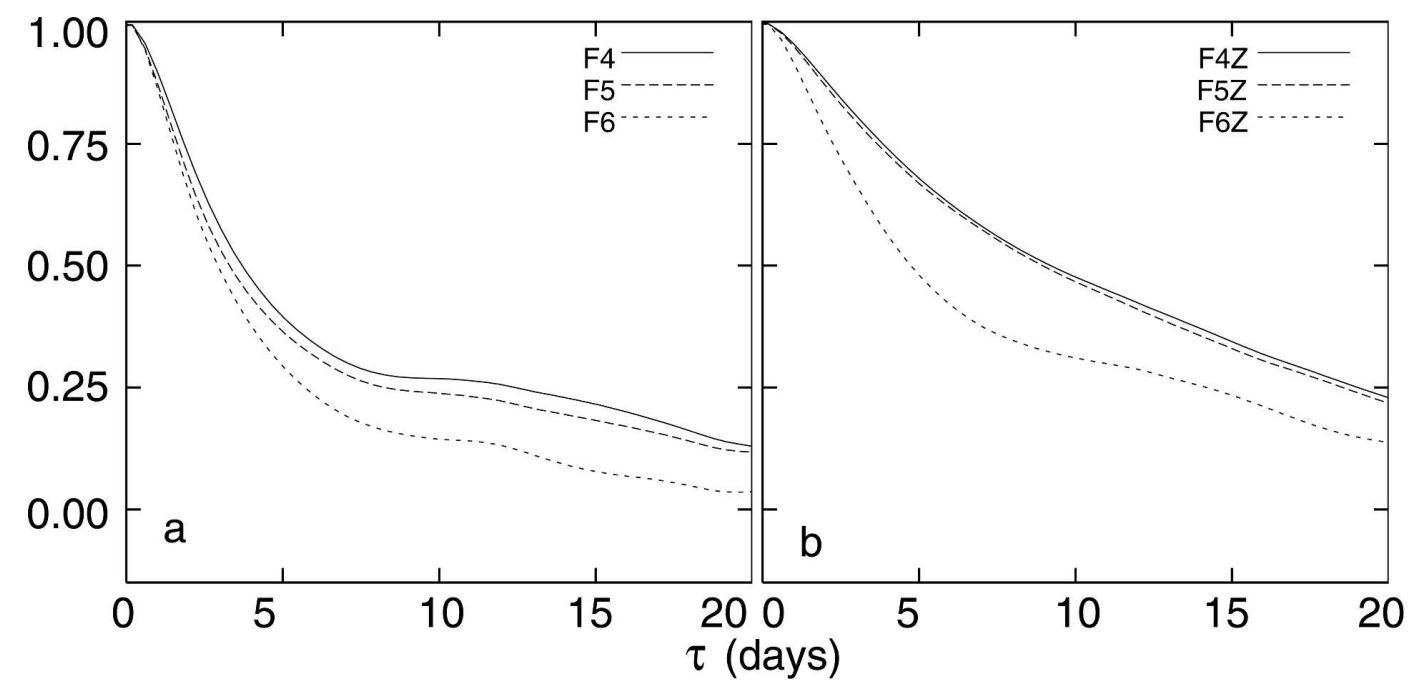

FIG. 5. (a) Autocorrelations of the wind terms $M_{w i}$ for the regions $F_{4}, F_{5}$, and $F_{6}$ in fall as a function of lag $\tau$ in days. Std dev is $6.8 \times 10^{5} \mathrm{Ha}$ s for $F_{4}, 6.1$ for $F_{5}$, and 1.7 for $F_{6}$. (b) Corresponding zonal domains $F_{4 z}, F_{5 z}$, and $F_{6 z}$. 


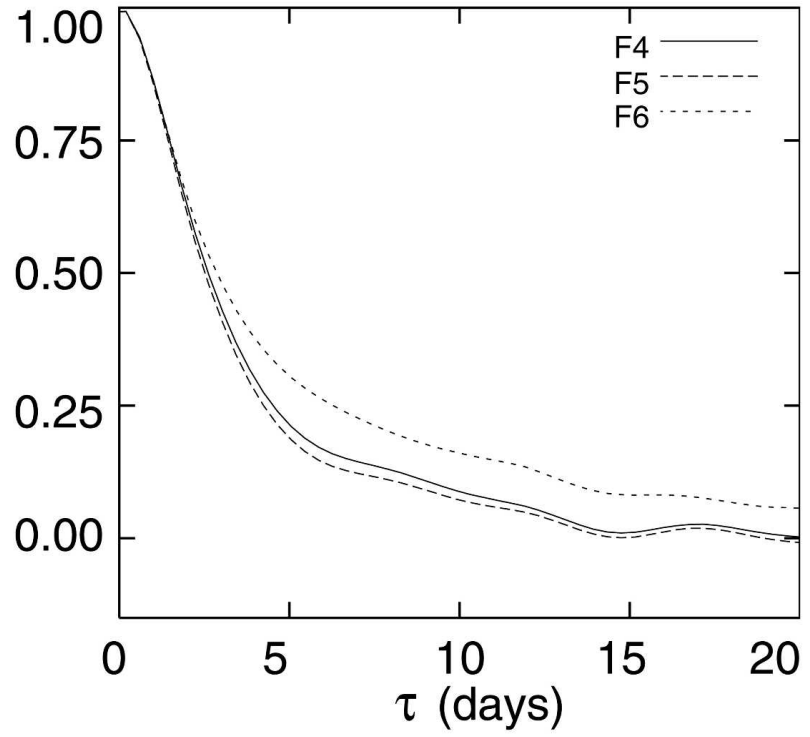

FIG. 6. Autocorrelations of the mass terms $M_{m i}$ for the regions $F_{4}, F_{5}$, and $F_{6}$ in fall as a function of lag $\tau$ in days. Std $\operatorname{dev}$ is $2.3 \times 10^{5} \mathrm{Ha}$ s for $F_{4}, 2.0$ for $F_{5}$, and 0.4 for $F_{6}$.

bations in the northern section $F_{6}$ have the longest memory. The corresponding autocorrelations for the low-pass-filtered time series (not shown) are quite similar to those in Figs. 5, 6. The correlations of the wind terms are $\sim 0.5$ after 5 days and reach a plateau with values $\sim 0.25$ at lags of 10 days and then decay slowly further on for $\tau>15$ days (not shown). The correlations for $M_{w 6}$ are lowest. The low-pass-filtered mass term autocorrelations are also quite similar to those in Fig. 6. They stay close to zero for $\tau \geq 10$ days. The autocorrelations of the wind terms for the zonal domains (Fig. $5 b)$ have a longer memory since zonal boundary fluxes and the pressure torques do not affect them [see (2.4)].

The correlation coefficients of the wind terms in $F_{2}$ and $F_{3}$ are small and negative but positive for the regional domains $F_{5}$ and $F_{6}$ (Table 3 ). The cross correlation of the mass terms is always positive. The wind terms in the southern section are negatively correlated with the northern mass terms. Strong zonal winds in the south imply low pressure in the north. It is the opposite with the mass terms in the south and the wind terms in the north.

Let us turn now to the tests of topographic instability. It is useful to formulate expectations before looking at the results. The angular momentum balance (2.4) reduces to

$$
\frac{d M_{w i}}{d t}=T_{o i}
$$

in a zonally periodic channel without friction and mass term variability. Thus
TABLE 3. Correlation coefficients of angular momentum terms in fall for the south/north area combinations $F_{2} / F_{3}$ and $F_{5} / F_{6}$.

\begin{tabular}{crc}
\hline \hline & $F_{2} / F_{3}$ & $F_{5} / F_{6}$ \\
\hline$M_{w} / M_{w}$ & -0.11 & 0.3 \\
$M_{m} / M_{m}$ & 0.26 & 0.68 \\
$M_{w} / M_{m}$ & -0.62 & -0.6 \\
$M_{m} / M_{w}$ & 0.55 & 0.57 \\
\hline
\end{tabular}

$$
\frac{d}{d \tau} C\left(T_{o i}, M_{w i} \mid \tau\right)=C\left(T_{o i}, T_{o i} \mid \tau\right)
$$

in this idealized case, which requires

$$
\int_{-\infty}^{+\infty} C\left(T_{o i}, T_{o i} \mid \tau\right) d \tau \sim 0 .
$$

It is clear that one cannot check (3.4) by looking at the observed autocovariance (see Fig. 4) because of uncertainties with respect to the data. Nevertheless, we expect that negative autocovariances of the torque prevail for $|\tau|>\tau_{o}$, where $\tau_{o}$ is the first zero-crossing. Correspondingly, $C\left(T_{o i}, M_{w i} \mid \tau\right)$ must be negative and decreasing for $\tau<-\tau_{o}$ and must increase in the interval $|\tau|<\tau_{o}$ to decrease again for larger lags. In particular, $C\left(T_{o i}, M_{w i} \mid \tau\right)$ is antisymmetric with respect to $\tau=0$ according to (3.3). There is no reason to believe that the observations near Greenland will fit closely (3.2), but (3.3) provides at least an orientation. In particular, the maximum increase

$$
\Delta C\left(T_{o i}, M_{i} \mid \tau\right)=\int_{-\tau_{o}}^{\tau_{o}} C\left(T_{o i}, T_{o i} \mid \tau\right) d \tau,
$$

possibly due to the mountain torque in the interval chosen, can be estimated from the data as displayed, for example in Fig. 4. This estimate has to be divided by the standard deviation of the mountain torque and by the factor $A_{i}$ to arrive at an estimate $\Delta \hat{u}_{i}$ of the maximum possible increase of the mean zonal wind. This maximum increase $\Delta \hat{u}_{i}$ can be compared to the observed increase of the zonal wind.

These maximum increases are listed in Table 4 for all seasons and for the analysis domains $F_{1}$ and $F_{4}$ as well as for their zonal extensions. The zero-crossings occur for $\tau=8-9$ days (see Fig. 4) except in winter when no zero-crossing is found. Nevertheless $\tau_{o}=9$ days is also accepted for December-February (DJF) for reasons of consistency. Also given are the standard deviations $\sigma_{\mathrm{i}}$ of the zonal wind terms as obtained from those of the wind terms by dividing by the factor $A_{1}$. It is seen that the torque-induced increase is comparable to $\sigma_{1}$ for $F_{1}$ 
TABLE 4. Maximum increase $\Delta \hat{u}_{i}$ of the zonal wind $\left(\mathrm{m} \mathrm{s}^{-1}\right)$ in the various analysis domains due to the mountain torque according to (3.5); also given in parentheses is the std dev of the zonal wind $\left(\mathrm{m} \mathrm{s}^{-1}\right)$ per domain as derived from that of the wind term.

\begin{tabular}{lrcrr}
\hline \hline & MAM & JJA & \multicolumn{1}{c}{ SON } & \multicolumn{1}{c}{ DJ } \\
\hline$F_{1}$ & $3.2(2.7)$ & $2.0(3.4)$ & $3.1(3.4)$ & $4.5(2.9)$ \\
$F_{1 z}$ & $0.8(2.2)$ & $0.6(2.7)$ & $0.8(3.2)$ & $1.2(2.7)$ \\
$F_{4}$ & $10.0(3.7)$ & $6.0(4.4)$ & $10.0(4.8)$ & $14.4(3.4)$ \\
$F_{4 z}$ & $1.7(2.6)$ & $1.1(3.0)$ & $1.7(3.7)$ & $2.5(2.7)$ \\
\hline
\end{tabular}

and larger than $\sigma_{4}$ for $F_{4}$. Torque events should be easily detectable in both cases provided the assumptions underlying (3.2) are satisfied. The impact of the torques is, of course, weaker in the zonal bands but should be detectable nevertheless.

Although (3.3) cannot be directly extended to the filtered time series, the estimate (3.5) nevertheless provides a gross expectation of the effects. There is one more difficulty with respect to the interpretation of the results. Although (3.2) suggests that the mountain torque is some kind of independent forcing, the surface pressure distribution at the mountain slopes in (2.1) depends itself on the zonal wind. The cross covariances of the torque and wind term to be displayed below reflect also the impact of the zonal wind on the torque and not only the "forcing" of $M_{w i}$ by the torques as implied by (3.2). Nevertheless, topographic instability should produce a signal that is smaller but of a similar order of magnitude as $\Delta \hat{u}_{i}$ [see (3.5)].

The cross correlations of the torques in the various domains with the related wind terms are displayed for $F_{1}$ and $F_{4}$ in Fig. 7 for all seasons. The correlations for the large domain $F_{1}$ are quite small and there is no obvious tendency for the wind term to grow in response to the torque except in spring where there is first a decrease from $\tau=-15$ to $\tau \approx-8$ days followed by an increase by $\sim 0.3$ until $\tau=6$ days. The maximum increase is $\Delta \hat{u}_{1} \sim 3.7 \mathrm{~m} \mathrm{~s}^{-1}$ (Table 4 ) while the observed increase of the zonal wind is about $1 \mathrm{~m} \mathrm{~s}^{-1}$ in about 10 days. That is a fairly weak signal. Correlations tend to be larger for $F_{4}$. In particular, there is an increase of the cross correlation by $\sim 0.4$ from $\tau=-2$ to $\tau=4$ days in spring. This corresponds with an increase of the mean

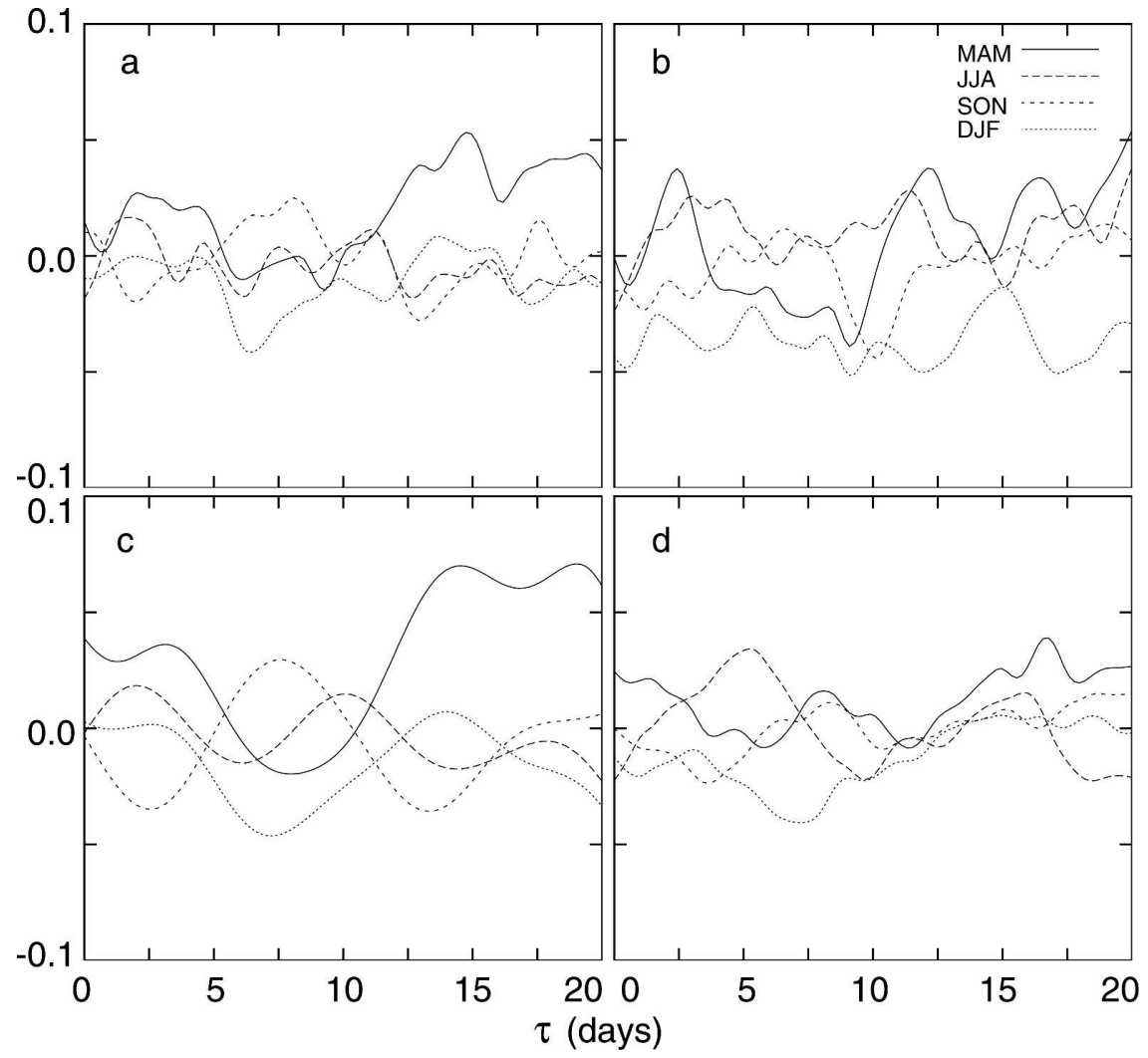

FIG. 7. Cross correlations of the Greenland mountain torque $T_{o 1}$ with the wind term for all seasons as a function of lag $\tau$ in days in (a) $F_{1}$, (b) $F_{4}$, (c) $F_{1}$ with filtered data, and (d) the zonal belt $F_{1 z}$. 
zonal velocity by $3.7 \mathrm{~m} \mathrm{~s}^{-1}$ within $\sim 10$ days. Accelerations are stronger above the mountains than in the wider domain $F_{1}$. This is an interesting result by itself that has, however, no implications for topographic instability because $F_{4}$ is too small.

The curves for the filtered time series (Fig. 7c) are essentially smoothed versions of those in Fig. 7a. In particular, it is seen even more clearly that the increase in spring should not be seen as an isolated event so that the correlations increase just by $\sim 0.1$ during a torque event. The switch to zonal domains (Fig. 7d) reveals that a "response" of the wind term to the torque may be found in spring and also in winter where there is first a decrease of the correlation coefficient but then an increase by $0.2-0.3$ within about 10 days. However, that corresponds with an increase of the zonal wind of less than $1 \mathrm{~m} \mathrm{~s}^{-1}$, a change without dynamical significance.

All other correlation functions have been computed as well. The result is essentially the same as in Fig. 7. There is little if any obvious reaction of the wind terms in the belts to the related torques. As an example, we show in Fig. 8 the cross correlation of the mountain torque in the northern belt with the wind term in $F_{3}$. It is only in fall that we see a distinct increase of the correlation by $\sim 0.2$ from $\tau \approx 0$ to $\tau=5$ days, which is, however, preceded by a similar decrease from $\tau=-10$ to $\tau=-5$ days so that the total gain of the wind term is rather small. The low-pass-filtered data yield an even weaker signal (not shown). The calculations for the zonal belts fail as well to provide a signal for dipole topographic instability.

Closeness to resonance is an important feature of the flow in the $\beta$-plane channel calculations of CDV, Pedlosky (1981), and Jin and Ghil (1990). On the sphere, resonance conditions can be specified as well for the wind profile $u \approx \cos \varphi$ of global superrotation but it would be difficult to select a representative scale for Greenland. Global resonance is, however, unimportant in any case because the impact of Greenland's mountain torque on the global flow is so small that any kind of related instability can be safely ruled out.

\section{b. Tibetan Plateau}

Weickmann (2003) regressed pressure and flows onto the mountain torque exerted by the total Eurasian massif, but the largest covariances are found near the plateau. An extended area of positive surface pressure anomalies is seen mainly north of the plateau, which extends then southward along the eastern low slope with increasing lag. Egger and Hoinka (2008) repeated and extended this analysis by including also the summer season and computing the covariances between the mountain torque and a rather large number of vari-

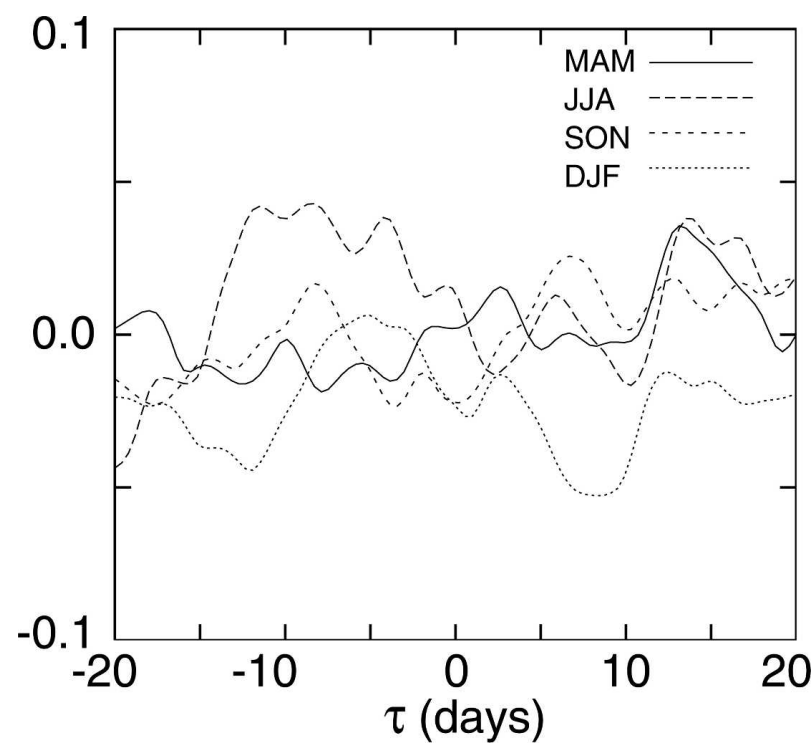

FIG. 8. Cross correlations of the northern Greenland mountain torque $\left(T_{o 3}\right)$ with the wind term in $F_{3}$ for all seasons.

ables. The Tibetan Plateau is not an ideal candidate for tests with respect to topographic instability because the atmospheric perturbation causing the torques are centered to the north of the Tibetan Plateau. It does not make sense to search for dipole instabilities in this situation.

The analysis domain $F\left(9^{\circ} \mathrm{N} \leq \varphi \leq 60.7^{\circ} \mathrm{N}, 49.5^{\circ} \mathrm{E} \leq\right.$ $\left.\lambda \leq 169.9^{\circ} \mathrm{E}\right)$ covers the plateau of Tibet and extends downstream almost to the date line. The overall response pattern for the Tibetan Plateau is similar to that found near Greenland. We present winter results (Fig. 9). The cross correlation of the zonal mean flow in the analysis domain with the mountain torque normalized by its standard deviation (Fig. 9) is always quite small. There is a minimum at $\tau=-1$ day, an increase until $\tau=2$ days, and constancy for larger lags. The response curve is reasonably asymmetric with respect to $\tau=0$ so that we see here a fairly clear response signal. The accelerations are, however, so small that this reaction is without dynamical significance. We have to stress that even the maximum $\Delta \hat{u} \approx 2 \mathrm{~m} \mathrm{~s}^{-1}$ is small in winter because a large analysis domain has to be chosen for this huge obstacle, which does, however, exert axial torques of the same order of magnitude as Greenland.

\section{Critique and conclusions}

The basic strategy of our tests is simple and appears to be robust. Topographic instability involves an interaction of the regional zonal mean flow with a mountain massif such that positive torques accelerate the zonal 


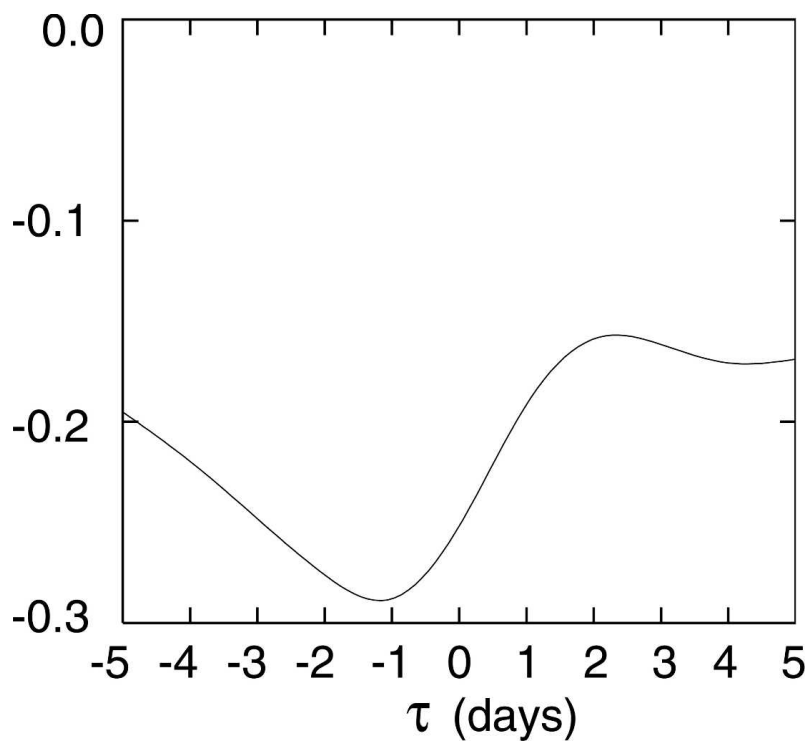

FIG. 9. Cross correlation of the Tibetan Plateau's normalized axial mountain torque with the zonal mean wind in winter (DJF). Std dev of the mountain torque is $10 \mathrm{Ha}$.

flow. The correlation of the regional wind term with the torque provides a basic indication. Of course, a clearcut positive response of the zonal flow would not prove that there is topographic instability but such a response would be seen as supporting evidence. We have not been able to detect the proper mean flow reaction either with respect to Greenland or for the plateau of Tibet. Greenland with its isolated topography and strong stationary wave component should have been an ideal candidate for such a test. However, the observations show clearly that the mountain torque is a minor contributor to the regional angular momentum budget. The acceleration due to the torque is hardly felt in the mountain region. This is true both for monopole tests where the torque and wind terms are evaluated for the total massif and for dipole tests where belts are selected in the north and also in the south. Results for the small domain $F_{4}$ are an exception in spring when the "response" of the zonal wind is relatively strong. The size of $F_{4}$ is, however, so small that conclusions with respect to topographic instability cannot be drawn. It is only in fall in the northern domain $F_{3}$ that zonal mean flow changes are large enough to be of eventual dynamical importance. There is, however, little further evidence that this is a case of topographic dipole instability.

Filtering of the data does not lead to new insights. There is no indication of topographic instability in the low-pass-filtered time series. Closeness to resonance is an important feature of the flow in the $\beta$-plane channel calculations of CDV, Pedlosky (1981), and Jin and Ghil (1990). On the sphere, resonance conditions can be specified as well for the wind profile $u \approx \cos \varphi$ of global superrotation, although it may be difficult to select a representative scale for Greenland. Global resonance is, however, unimportant in any case because the impact of Greenland's mountain torque on the global flow is so small that any kind of related instability can be safely ruled out.

We have to keep in mind that our approach is statistical and favors the type of events that occur most often. Assume that a few examples of topographic instability occur during a season. In principle, such events could be detected by performing a regional angular momentum budget according to (2.4) on a day-by-day basis. These rare events would have little impact on the correlations of the torque and wind terms as displayed above. Our results do not, therefore, rule out topographic instability for Greenland and the Tibetan Plateau. What is excluded is the climatic relevance of this instability. This result is not in conflict with Frederiksen and Bell (1987), because the instabilities found by these authors allow for variations of the regional mean flow but do not imply a strong local response of the zonal mean flow to the torques.

Nevertheless, one may wonder why we did not conduct such daily analyses. It is, however, a main problem with such efforts that the mountain torque is hardly ever a leading term in the budget, so that its impact on the regional wind term is not dominant. To demonstrate this point (see also Egger and Hoinka 1992) we assume in (2.4) inviscid $f$-plane geometry for the sake of simplicity. Let us introduce the standard separation of the meridional wind component

$$
v=\left(\rho f_{o}\right)^{-1} \frac{\partial p}{\partial x}+v_{\mathrm{ag}}
$$

with ageostrophic meridional wind $v_{\mathrm{ag}}$. Since

$$
\begin{aligned}
M_{w} & =\int_{V} \rho u a \cos \varphi_{o} d V, \\
M_{m} & =\int_{V} \rho f_{o} a \cos \varphi_{o} y d V, \\
\frac{d M_{m}}{d t} & \approx-\int \rho f_{o} a \cos \varphi_{o} v d V
\end{aligned}
$$

in this case, the geostrophic part of the Coriolis term cancels in (2.4) the pressure torque and the mountain torque so that we are left with

$$
\begin{aligned}
\frac{d}{d t} M_{w}= & - \text { boundary fluxes of } \rho u \\
& +\int \rho f_{o} a \cos \varphi_{o} v_{\mathrm{ag}} d V
\end{aligned}
$$


The mountain torque is no longer part of the budget. It is only the zonal momentum fluxes through the boundary and the ageostrophic meridional mass flux that affect the wind term. That does, of course, not imply that the mountain torque does not affect the momentum of the regional flow, but since $\left|v_{\mathrm{ag}}\right| \ll\left|v_{\mathrm{g}}\right|$ in most cases we have a cancellation of large terms that makes it difficult if not impossible to demonstrate the impact of the mountain torque on $M_{w}$ by case studies (e.g., Czarnetzki 1997). The ideal test case would have no eastwest pressure difference between the boundaries and no mean meridional motion. In that case, only the boundary fluxes compete with the mountain torque. One would have to search for situations of this type. On the other hand, meridional motion is strong during torque events. Our statistical approach circumvents these problems because we just correlate the mountain torque with the wind term. The negative outcome of our tests indicates that the ageostrophic meridional mass transport in the Greenland domain is essentially uncorrelated with the mountain torque as are the boundary fluxes.

The switch to the zonal domains excludes the large zonal boundary fluxes. Moreover, there is no pressure torque so (4.5) becomes

$$
\begin{aligned}
\frac{d M_{w}}{d t}= & - \text { meridional boundary fluxes of } \rho u \\
& +\int \rho f_{o} a \cos \varphi_{o} v_{\mathrm{ag}} d V+T_{o} .
\end{aligned}
$$

One would hope to see the impact of the mountain torque more clearly in this configuration. There is, however, the drawback that the response volume is quite large for zonal belts. Correspondingly, the response of the zonal wind is small if it can be found at all.

In conclusion we may state that there is no evidence for topographic instability at the statistical level of this paper. This negative result corroborates the findings of Revell and Hoskins (1984). We have to stress, however, that we considered only Greenland and the Tibetan Plateau. The Andes Mountains or the North American massifs may turn out to be regions with more positive results.

Acknowledgments. Valuable suggestions by the reviewers led us to admit zonal belts as test domains and to perform the analysis also with filtered data.

\section{REFERENCES}

Blackmon, M. L., and N.-C. Lau, 1980: Regional characteristics of the Northern Hemisphere wintertime circulation: A comparison of the simulation of a GFDL general circulation model with observations. J. Atmos. Sci., 37, 497-514.
Charney, J., and J. DeVore, 1979: Multiple flow equilibria in the atmosphere and blocking. J. Atmos. Sci., 36, 1205-1216.

— ria and propagating planetary waves in baroclinic, orographically forced, planetary wave systems. J. Atmos. Sci., 37, 11571176.

Czarnetzki, A., 1997: Regional mountain torque estimates over the Rocky Mountains in lee cyclones. J. Atmos. Sci., 54, 19861997.

Dickey, J., M. Ghil, and S. Marcus, 1991: Extratropical aspects of the 40-50 day oscillation in length-of-day and atmospheric angular momentum. J. Geophys. Res., 96 (D12), 22 643-22 658.

Egger, J., 2003: A modified quasigeostrophic equation for barotropic mean flow over topography. Meteor. Z., 12, 43-46.

— planetary flow. Quart. J. Roy. Meteor. Soc., 107, 299-312.

_ , and K.-P. Hoinka, 1992: Fronts and orography. Meteor. Atmos. Phys., 48, 3-36.

$\longrightarrow$, and - 2006: Dynamics of atmospheric regression patterns: Regional mountain torque events. J. Atmos. Sci., 63, 1467-1482.

- , and - 2008: Mountain torque events at the Tibetan Plateau. Mon. Wea. Rev., in press.

Frederiksen, C., and J. S. Frederiksen, 1991: Flow over topography and instability on a sphere. J. Atmos. Sci., 48, 2411-2425.

Frederiksen, J. S., 1982: Eastward and westward flows over topography in nonlinear and linear barotropic models. J. Atmos. Sci., 39, 2477-2489.

_ zonal solutions for flow over topography. Geophys. Astrophys. Fluid Dyn., 35, 173-207.

— baroclinic, barotropic and topographic instability. J. Atmos. Sci., 44, 2200-2218.

Fyfe, J., and J. Derome, 1986: A barotropic stability study of free and forced planetary waves. J. Atmos. Sci., 43, 2162-2182.

Ghil, M., and S. Childress, 1987: Topics in Geophysical Fluid Dynamics: Atmospheric Dynamics, Dynamo Theory, and Climate Dynamics. Springer-Verlag, 485 pp.

Jin, F.-F., and M. Ghil, 1990: Intraseasonal oscillations in the extratropics: Hopf bifurcation and topographic instabilities. $J$. Atmos. Sci., 47, 3007-3022.

Lott, F., A. Robertson, and M. Ghil, 2004: Mountain torques and Northern Hemisphere low-frequency variability. Part I: Hemispheric aspects. J. Atmos. Sci., 61, 1259-1271.

Metz, W., 1985: Wintertime blocking and mountain forcing of the zonally-averaged flow: A cross-spectral time series analysis of observed data. J. Atmos. Sci., 42, 1880-1892.

Pedlosky, J., 1981: Resonant topographic waves in barotropic and baroclinic flows. J. Atmos. Sci., 38, 2626-2641.

Revell, M., and B. Hoskins, 1984: Orographically induced Rossby wave instabilities. J. Atmos. Sci., 41, 51-67.

Tung, K., and A. J. Rosenthal, 1985: Theories of multiple equilibria-A critical reexamination. Part I: Barotropic models. $J$. Atmos. Sci., 42, 2804-2819.

Uppala, S. M., and Coauthors, 2005: The ERA-40 re-analysis. Quart. J. Roy. Meteor. Soc., 131, 2961-3012.

Weickmann, K., 2003: Mountains, the global frictional torque, and the circulation over the Pacific-North American region. Mon. Wea. Rev., 131, 2608-2622.

_ W. Robinson, and C. Penland, 2000: Stochastic and oscillatory forcing of global atmospheric angular momentum. $J$. Geophys. Res., 105 (D12), 15 543-15 558. 